Jpn. J. Med. Sci. Biol., 51, Suppl. 1, S91-S100, 1998

\title{
Enteropathogenic E. coli Interactions with Host Cells
}

B. Brett Finlay ${ }^{1)}$ and Akio Abe ${ }^{1,2)}$

1) Biotechnology Laboratory and the Departments of Biochemistry \& Molecular Biology, and Microbiology \& Immunology, University of British Columbia, Vancouver, B.C. Canada V6T-1Z3

2) Department of Bacteriology, The Kitasato Institute, Minato-ku, Tokyo 108-8642, Japan

\section{SUMMARY}

Enteropathogenic E. coli (EPEC) interacts with intestinal epithelial cells, causing diarrhea and associated diseases. This pathogen binds to epithelial cells using sophisticated mechanisms that exploit existing epithelial signal transduction pathways and host cytoskeletal components, ultimately resulting in the bacterium resting upon a pedestal on host cell surfaces. Recent data indicates that similar mechanisms occur in vivo. EPEC interactions with host cells illustrate several principles of pathogenesis that are used by bacteria that interact with mammalian host cells.

\section{EPEC-Mediated Disease}

\section{INTRODUCTION}

Escherichia coli is an extremely versatile pathogen. In addition to being a member of the normal intestinal flora, $E$. coli also causes bladder infections, meningitis, and diarrhea. Diarrheagenic $E$. coli include at least five types of $E$. coli, which cause various symptoms ranging from cholera-like diarrhea to extreme colitis. Each type of diarrheagenic E. coli possesses a particular set of virulence factors, including specific adhesins, invasins, and/or toxins, which are responsible for causing a specific type of diarrhea. One of these groups, enteropathogenic E. coli (EPEC), is a predominant cause of infant diarrhea worldwide. In addition to isolated outbreaks in daycares and nurseries in developed countries, EPEC poses a major endemic health threat to young children ( $<6$ months) in developing countries, where it has a high mortality rate. Worldwide, EPEC is the leading cause of bacterial mediated diarrhea in children, and it has been 
estimated that EPEC kills several hundred thousand children per year worldwide. EPEC disease is characterized by watery diarrhea of varying severity, while vomiting and fever often accompany fluid loss.

Despite the significance of EPEC mediated disease, little is known about how this pathogen actually causes disease (3). Unlike other $E$. coli diarrheas such as enterotoxigenic $E$. coli, EPEC diarrhea is not mediated by a toxin. Instead, EPEC binds to intestinal surfaces of the small bowel. A characteristic histological lesion, called the attaching and effacing (A/E) lesion, occurs (3). A/E lesions are marked by dissolution of the intestinal brush border surface and loss of epithelial microvilli (effacement) at the sites of bacterial attachment. Once bound, EPEC reside upon a cup-like projection or pedestal upon which adherent bacteria reside. It has been shown that underlying this pedestal in the epithelial cell are several cytoskeletal components, including actin, alpha-actinin, ezrin, talin, and myosin light chain (8). Formation of the $\mathrm{A} / \mathrm{E}$ lesion appears to be responsible for fluid secretion and diarrhea, although mechanistically this remains to be proven. It has been suggested that disruption of the brush border and microvilli may be responsible for diarrhea. Although EPEC can enter (invade) tissue culture cells (2), it does not normally cause invasive disease, and rarely penetrates the intestinal barrier.

EPEC belongs to a group of pathogenic organisms that form A/E lesions, including enterohemorrhagic E. coli (EHEC), several EPEC-like animal pathogens that cause disease in rabbits (REPEC), dogs, pigs (PEPEC), etc, Citrobacter rodentium, Hafnia alvei, and possibly even Helicobacter pylori. These organisms all cause cytoskeletal rearrangement and pedestal formation on relevant host epithelial cells. EHEC, which causes enteric colitis ("hamburger disease") can also cause haemolytic uraemic syndrome in approximately $10 \%$ of cases. EHEC possess all of the EPEC virulence factors needed for A/E lesion formation, but has an additional shiga-like toxin, which contributes to its increased pathogenesis.

\section{Mechanisms of EPEC Pathogenicity}

Initial Adherence. Recently, significant progress has been made in defining the bacterial and host factors involved in formation of attaching and effacing lesions. Initial bacterial adherence is dependent on the presence of a 55-70 MDa plasmid that is common to EPEC strains. This process is mediated by a plasmid encoded bundle forming pilus (BFP) (reviewed in (3)). Mutants in EPEC that are defective in initial adherence produce fewer $\mathrm{A} / \mathrm{E}$ lesions on epithelial cells, but these lesions are indistinguishable from those caused by parental EPEC. Instead of EPEC, the initial adherence of rabbit EPEC strains, RDEC-1 and REPEC O103, is 
mediated by $\mathrm{AF} / \mathrm{R} 1$ and $\mathrm{AF} / \mathrm{R} 2$ fimbriae, respectively. These differences in the initial adherence might relate to their host specificity.

Signal transduction. When EPEC interact with cultured epithelial cells, several signal transduction pathways are activated in the epithelial cells, including the release of the eukaryotic secondary messengers, IP 3 , and intracellular calcium $(5,9)$. EPEC binding to cultured epithelial cells also causes tyrosine phosphorylation of a $90 \mathrm{kDa}$ protein found in the membranes of epithelial cells, Tir (formerly $\mathrm{Hp90}$ ), which is not phosphorylated in uninfected cultured cells $(16,17)$. Addition of tyrosine kinase inhibitors inhibit the phosphorylation of Hp90 and EPEC uptake into epithelial cells. It appears that Tir phosphorylation precedes $\mathrm{IP}_{3}$ fluxes and cytoskeletal rearrangements (9). A second wave of signals occur as intimin (see below) mediates intimate adherence to host cells (13). These signals include tyrosine phosphorylation of phosholipase $\mathrm{C}$ gamma.

All of the EPEC genes known to be involved in A/E formation (except the plasmid encoded regulator, per) are found within a unique contiguous region in the EPEC chromosome called LEE, for locus of enterocyte attachment (15). Several bacterial loci have been identified within this region that are involved in activating epithelial signal transduction (reviewed in (3)). Strains containing mutations in genes encoding the secreted proteins EspA (E. coli secreted protein A), EspB (formerly EaeB), or EspD, do not stimulate signal transduction or cytoskeletal rearrangement. Strains cured of the EPEC virulence plasmid are still capable of activating epithelial signal transduction pathways and organizing the underlying cytoskeletal structure, although their efficiency at these events is significantly decreased, presumably due to the loss of the plasmid encoded bundle-forming pilus and the plasmid encoded positive regulator, Per. Much of the LEE locus encodes a type III secretion apparatus (10). This locus is needed to secrete EspA, EspB, and EspD (14), and thus mutations in this secretion locus are also unable to trigger signals in host cells, due to the lack of secreted effector molecules.

Such type III secretion systems are used by several bacterial pathogens to export virulence factors out of the bacteria and into contact with mammalian cells $(6,7)$. Examples include the invasion systems of Shigella and Salmonella species, the Yersinia secreted proteins (YOPs) including a tyrosine phosphatase which enters phagocytic cells, and harpins from plant pathogens. Collectively, this information indicates that EPEC secretes at least three molecules (EspA, EspB, and EspD) that are critical for activating signal transduction and cytoskeletal rearrangement in epithelial 
cells, and EPEC has a specialized secretion system for exporting these molecules.

Intimate adherence. Intimin is the product of a bacterial chromosomal locus, eaeA, and is a $94 \mathrm{kDa}$ EPEC outer membrane protein that is needed for intimate adherence (11). eaeA mutants form immature A/E lesions and do not organize phosphotyrosine proteins and cytoskeletal components beneath adherent bacteria, although epithelial signal transduction is still activated (16). Intimin appears to participate in reorganization of the underlying host cytoskeleton after other bacterial factors stimulate epithelial signal transduction. Although cloned intimin in non-pathogenic $E$. coli does not mediate adherence (11), if an eaeA defective strain of EPEC is added to epithelial cells prior to addition of the cloned intimin expressed in E. coli HB101, bacteria expressing the cloned intimin now adhere to epithelial cells (17). However, if EPEC mutants that are defective for stimulating signal transduction (such as $e s p B$ ) are used to preinfect monolayers, organisms containing the cloned intimin do not adhere. Although we originally interpreted these results to indicate that EPEC needed to activate host signalling prior to intimin binding to host cell surfaces (17), we now know that these pathogens use an even more sophisticated adherence mechanism that involves injecting their own receptor into host cells (12).

As discussed above, EPEC triggers tyrosine phosphorylation of Tir, a $90 \mathrm{kDa}$ membrane-located protein that localizes beneath adherent organisms at the tip of extended pseudopods (17). This protein interacts with intimin, and is an intimin receptor. If EPEC is added to epithelial cells and then removed by detergent extraction, Tir remains associated with the bacteria; however, if an eaeA mutant is used, Tir is not isolated with the bacteria (17). Tir is also coimmunoprecipitated with the intiminmaltose binding protein fusion peptide, but only if epithelial cells are preintected by EPEC strains. Intimin fused to MBP also binds directly and specifically to Tir when HeLa cell membrane extracts from eaeApreinduced cells are separated by PAGE, renatured, and hybridized with MBP/Int. MBP/Int does not bind to membrane extracts from uninfected monolayers, or those infected with signaling mutants such as esp $B$.

We have recently identified $\operatorname{Tir}(12)$ and, surprisingly, it is a bacterial protein that is inserted into the host membrane by the Esp products which utilize the type III secretion system. Tir is secreted as a $78 \mathrm{kDa}$ unphosphorylated protein by EPEC. Once inserted into the host membrane, it is tyrosine phosphorylated and its apparent molecular weight shifts to $90 \mathrm{kDa}$. Unlike the Esp proteins, its amino-terminal methionine 
is missing from the bacterial secreted protein, and we are currently unsure whether it is secreted directly by the type III system. It is clear that the Esp products are needed to insert Tir into host membranes.

The role of tyrosine phosphorylation of Tir is also unclear. Using in vitro systems, we showed that the bacterial secreted form of Tir (unphosphorylated) bound intimin in a dose dependant, saturatable, and competitive manner (12). We are currently mutagenizing Tir to define the exact role (if any) of tyrosine phosphorylation.

Cytoskeletal Rearrangement and Pedestal Formation. As described above, the attaching/effacing (A/E) lesion (or pedestal) formed by EPEC upon association with epithelial cells is associated with the assembly of highly organized cytoskeletal structures in the epithelial cells immediately beneath adherent bacteria (3). Although this pedestal usually raises the bacterium slightly above the epithelial cell surface, EPEC can trigger extended pseudopod formation, with projections extending up to 10 microns above the epithelial cell surface with the bacteria located extracellularly at the tip of these extensions (17). The stalk of these extended pseudopods contains polymerized actin, while Tir is localized only at the tip of these structures beneath EPEC. Extended pedestals are not seen when strains containing mutations in eaeA, espB, or type III loci are used, reinforcing the linkage between signal transduction events and cytoskeletal rearrangement.

It appears the product of the eaeA gene, intimin, is critical for organizing cytoskeletal rearrangements. eaeA mutants trigger signals in epithelial cells and cause generalized actin accumulation near adherent organisms, but are unable to organize the cytoskeleton into defined structures that lead to pedestal and extended pseudopod formation. Additionally, they do not invade epithelial cells, even if complemented with signal transduction defective EPEC mutants. Further support for the role of intimin comes from experiments performed with the cloned EPEC intimin expressed in non-pathogenic $E$. coli HB101. If EPEC containing a defective eaeA gene are first added to epithelial cells followed by strains containing cloned intimin, only HB101 harboring intimin, but not the eaeA mutant, organize the cytoskeleton into pedestals and extended pseudopods (17). This indicates that intimin molecules direct the final condensation and organization of the host cytoskeleton from their outer membrane location on the adherent $E$. coli. Tir also probably plays a significant role in organizing the host cytoskeleton, in addition to mediating intimin binding. It probably serves as a bridge, linking intimin in the bacterial outer membrane to the host cytoskeleton on the other side of the epithelial cell 
membrane.

Production of Diarrhea? Despite our increasing knowledge of the bacterial factors and host molecules that mediate EPEC interactions with epithelial cells, the actual molecular mechanisms that cause diarrhea remain undefined. EPEC strains lacking intimin are significantly decreased in their ability to cause diarrhea in human volunteers (4). It is probable that one or more of the events associated with formation of attaching and effacing lesions also causes diarrhea. In addition to the morphological rearrangements that occur on the apical surface of epithelial cells, EPEC also causes a large decrease in transepithelial resistance in polarized Caco2 epithelial cell monolayers (1). Mutants defective for signal transduction and eaeA mutants do not cause this loss in transepithelial resistance, indicating that this process is linked to these events. It is possible that such transepithelial disruptions occur in vivo, which would lead to ionic imbalances and possibly diarrhea.

By using whole cell patch clamping technology, we have found that EPEC causes a significant depolarization of individual $\mathrm{HeLa}$ cells. Although eaeA mutants still caused depolarization, espB and type III mutants do not depolarize cells, indicating that EPEC secreted proteins which affect epithelial signaling are needed for these events. Occurrence of such a process in the gut would reduce the electrochemical gradient available for sodium ion absorption from the gut lumen, thereby contributing to ionic imbalance, fluid loss, and diarrhea.

Extending in vitro to in vivo observations. Given the human specific nature of EPEC, it is difficult to test whether the various events that occur as EPEC interacts with cultured cells actually occur in vivo. However, we have begun to use a naturally occurring rabbit EPEC infection model to study these events in vivo. Using young rabbits infected orally with enteropathogenic $E$. coli serotype O103 (REPEC O103), we have found that several events similar to those seen in tissue culture also occur in vivo. For example, extended pedestals and intimate attachment are seen using scanning and transmission electron microscopy. Additionally, the results of confocal laser scanning microscopy studies showed that cytoskeletal actin rearrangements occurred beneath adherent REPEC O103. This strain extensively disrupts host microvilli and possibly disrupts the epithelial barrier. We have also found that mutations in esp $A$ and espB are avirulent in this model. Although they still adhere to intestinal surfaces, they are unable to cause A/E lesions, nor do they affect intestinal microvilli in any discernable way. These results indicate that indeed the 
I. Initial adherence by pili or fimbriae

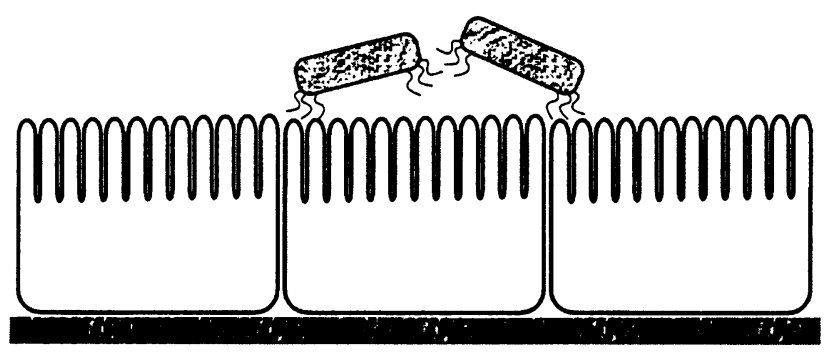

Potential Bacterial factors involved in process

- BFP (EPEC 0127)

- AF/R1 (RDEC-1 015)

- AF/R2 (REPEC 0103)

II. Signal transduction and microvilli effacement

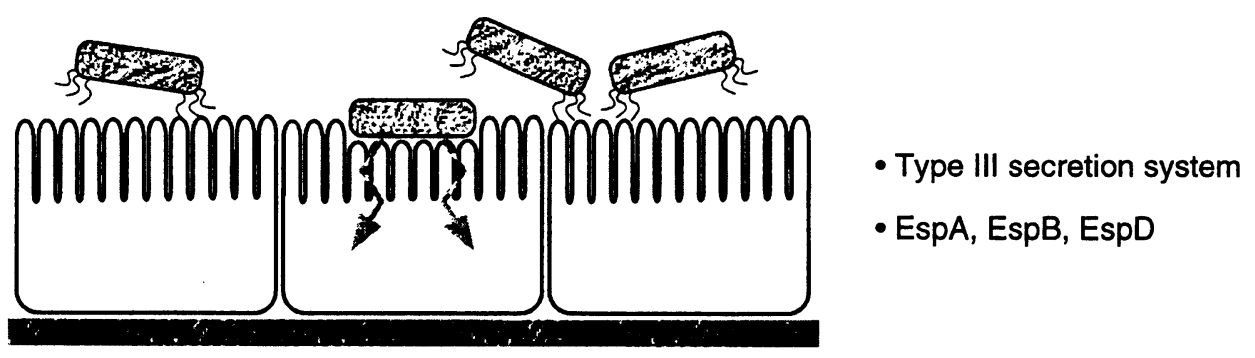

III. Cytoskeletal rearrangement and, intimate adherence, pedestal formation

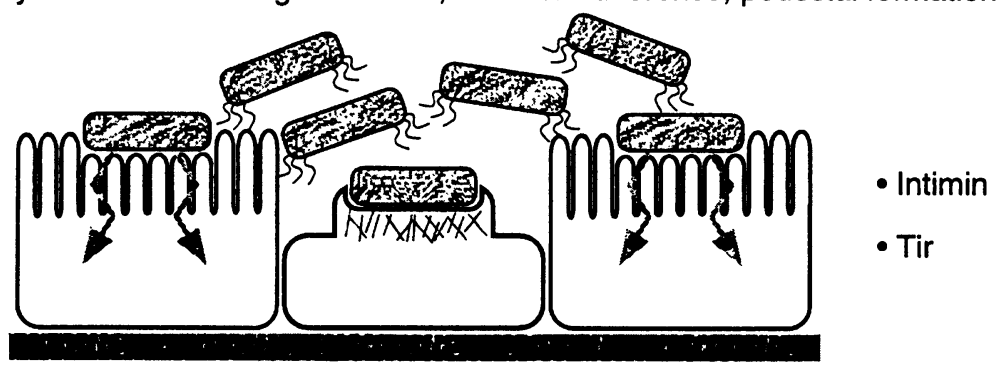

IV. Disruption of intestinal surface by continued AVE lesion

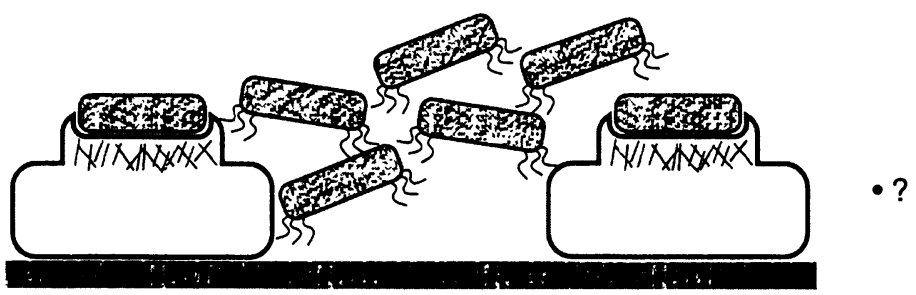

Fig. 1. A schematic diagram of EPEC interactions with host cells. 
LEE locus encodes virulence factors that are needed for disease. Based on these results, we present a possible model of production of diarrhea by EPEC infection (Fig. 1). Initial adherence to the epithelial surface is mediated by pili or fimbriae. The first step is followed by triggering of host signal transduction pathways and microvilli effacement. The type III secretion system and Esp proteins are necessary for this step. In the third step, the bacteria cause cytoskeletal rearrangements and formation of pedestal-like structures beneath adherent bacteria due to binding of intimin and Tir. Finally bacteria disrupt the intestinal surface by continued $\mathrm{A} / \mathrm{E}$ lesions. Some effectors similar to the Yersinia YOPs might be involved in this final step. Employment of this animal infection model will be extremely useful in the future to confirm additional events, and to begin to probe the mechanisms involved in diarrhea production, including inflammation.

\section{CONCLUSIONS}

It is becoming apparent that many successful bacterial pathogens are capable of exploiting host cell processes and functions. By activating host cell signal transduction, rearranging the host cytoskeleton, and inserting its own receptor, EPEC mediates intimate adherence to epithelial cells. These events are orchestrated by a type III secretion system and secreted effector molecules that are clustered within a pathogenicity island on EPEC's chromosome, along with the factors needed for intimate adherence (Tir and intimin). By employing a relevant animal model, it appears that similar events occur in vivo and are related to production of disease. Collectively, these results suggest intimate interactions with epithelial cells play a key role in pathogenesis for these organisms and ultimately production of disease.

\section{ACKNOWLEDGEMENTS}

Work in my laboratory is supported by the Medical Research Council of Canada and a Howard Hughes International Research Scholar award. BBF is a MRC Scientist.

\section{REFERENCES}

1. Canil, C., I. Rosenshine, S. Ruschkowski, M.S. Donnenberg, J.B. Kaper, and B.B. Finlay. (1993): Enteropathogenic Escherichia coli decreases the transepithelial electrical resistance of polarized epithelial monolayers. Infect. Immun. $61:$ 2755-2762.

2. Donnenberg, M.S., A. Donohue-Rolfe, and G.T. Keusch. (1990): A comparison of HEp-2 cell invasion by enteropathogenic and enteroinvasive Escherichia coli. FEMS Microbiol. Letts. 57 : 83-86. 
3. Donnenberg, M.S., J.B. Kaper, and B.B. Finlay. (1997): Interactions between enteropathogenic Escherichia coli and host epithelial cells. Trends Microbiol. $5: 109-114$.

4. Donnenberg, M.S., C.O. Tacket, S.P. James, G. Losonsky, J.P. Nataro, S.S. Wasserman, J.B. Kaper, and M.M. Levine. (1993): Role of the eaeA gene in experimental enteropathogenic Escherichia coli infection. J. Clin. Invest. 92 : 1412-1417.

5. Dytoc, M., L. Fedorko, and P.M. Sherman. (1994): Signal transduction in human epithelial cells infected with attaching and effacing Escherichia coli in vitro. Gastroenterology. $106: 1150-1161$.

6. Finlay, B.B. and P. Cossart. (1997): Exploitation of mammalian host cell functions by bacterial pathogens. Science. $276: 718-725$.

7. Finlay, B.B. and S. Falkow. (1997): Common themes in microbial pathogenicity. II. Molecular Biology and Microbiological Reviews. 61: 136169.

8. Finlay, B.B., I. Rosenshine, M.S. Donnenberg, and J.B. Kaper. (1992): Cytoskeletal composition of attaching and effacing lesions associated with enteropathogenic Escherichia coli adherence to HeLa cells. Infect. Immun. $60: 2541-2543$.

9. Foubister, V., I. Rosenshine, and B.B. Finlay. (1994): A diarrheal pathogen, enteropathogenic Escherichia coli (EPEC), triggers a flux of inositol phosphates in infected epithelial cells. J. Exp. Med. 179 : 993-998.

10. Jarvis, K.G., J.A. Giron, A.E. Jerse, T.K. McDaniel, M.S. Donnenberg, and J.B. Kaper. (1995): Enteropathogenic Escherichia coli contains a putative type III secretion system necessary for the export of proteins involved in attaching and effacing lesion formation. Proc. Nat'l. Acad. Sci. USA. 92 : 7996-8000.

11. Jerse, A.E., J. Yu, B.D. Tall, and J.B. Kaper. (1990): A genetic locus of enteropathogenic Escherichia coli necessary for the production of attaching and effacing lesions on tissue culture cells. Proc. Nat'1. Acad. Sci. USA. 87 : 7839-7843.

12. Kenny, B., R. Devinney, M. Stein, D.J. Reinscheid, E.A. Frey, and B.B. Finlay. (1997): Enteropathogenic $E$. coli (EPEC) transfers its receptor for intimate adherence into mammalian cells. Cell. $91: 511-520$.

13. Kenny, B. and B.B. Finlay. (1997): Intimin-dependent binding of enteropathogenic Escherichia coli to host cells triggers novel signaling events, including tyrosine phosphorylation of phospholipase $\mathrm{C}$ gamma. Infection and Immunity. $65: 2528-2536$.

14. Kenny, B. and B.B. Finlay. (1995): Protein secretion by enteropathogenic Escherichia coli is essential for transducing signals to epithelial cells. Proc. Nat'l. Acad. Sci. USA. 92 : 7991-7995.

15. McDaniel, T.K., K.G. Jarvis, M.S. Donnenberg, and J.B. Kaper. (1995): A genetic locus of enterocyte effacement conserved among diverse enterobacterial pathogens. Proc. Nat'l. Acad. Sci. USA. 92 : 1664-1668.

16. Rosenshine, I., M.S. Donnenberg, J.B. Kaper, and B.B. Finlay. (1992): Signal transduction between enteropathogenic Escherichia coli (EPEC) and epithelial cells: EPEC induces tyrosine phosphorylation of host cell proteins to initiate 
cytoskeletal rearrangement and bacterial uptake. EMBO J. 11:3551-3560.

17. Rosenshine, I., S. Ruschkowski, M. Stein, D.J. Reinscheid, S.D. Mills, and B.B. Finlay. (1996) A pathogenic bacterium triggers epithelial signals to form a functional bacterial receptor that mediates actin pseudopod formation. EMBO J. $15: 2613-2624$. 